\title{
Pleural Malignant Mesothelioma Causing Cord Infiltration Through the Nerve Root
}

\author{
-Case Report-
}

\author{
Hidehiro OKURA, Yasuo SUgA, Osamu AKIYAMA, Kentaro KUdO, \\ Satoshi Tsutsumi, Yusuke ABE, Yukimasa YASUMOTO, \\ Masanori ITO, Hiroshi IZUMI*, and Kazu SHIOMI** \\ Departments of Neurosurgery and \\ ${ }^{* *}$ General Thoracic Surgery, Juntendo Urayasu Hospital, Urayasu, Chiba; \\ ${ }^{*}$ Department of Human Pathology, Juntendo University School of Medicine, Tokyo
}

\begin{abstract}
A 61-year-old man presented with a rare pleural malignant mesothelioma of the spine manifesting as progressive weakness of the bilateral lower extremities, numbness in the body and both legs, and dysfunction of the bladder and bowel. He had previous occupational exposure to asbestos while working at a car repair shop and had undergone right panpleuropneumonectomy under a diagnosis of sarcomatous type mesothelioma in the right pleural space. Magnetic resonance imaging of the spine with gadolinium showed an enhanced intramedullary tumor at the $\mathrm{T} 4$ level. Operative findings disclosed the clouded and swollen right posterior nerve root, and the pial surface was covered by clouded arachnoid-like membrane. The removed part of the $\mathrm{T} 4$ posterior nerve root and intramedullary tumor revealed malignant mesothelioma with invasion spreading along the posterior nerve root. He died of respiratory failure 3 months after the diagnosis. This case shows that spinal metastasis must be considered if a patient with pleural malignant mesothelioma shows neurological worsening and neuroimaging shows an abnormal lesion in the thoracic spinal cord. However, the patient's neurological condition is very difficult to improve in the presence of spinal cord infiltration.
\end{abstract}

Key words: pleural malignant mesothelioma, intramedullary metastasis, nerve root

\section{Introduction}

Malignant mesothelioma is a neoplasm usually arising from the mesothelial surfaces of the pleural and peritoneal cavities, pericardium, or tunica vaginalis, and $80 \%$ of cases arise from the pleura. ${ }^{15)}$ Pleural malignant mesothelioma is an uncommon neoplasm of the pleural epithelium, strongly related to inhalational exposure to asbestos, ${ }^{9}$ with a mean delay between exposure and presentation of 29 years for factory workers. ${ }^{17)}$ The most frequent manifestations are dyspnea, cough, and chest pain. Three histological types are known: epithelioid (45\%), sarcomatoid $(24 \%)$, and biphasic (31\%). ${ }^{17)}$ Pleural malignant mesothelioma is characterized by the tendency to spread along preexisting tissue planes. ${ }^{10)}$ In general, pleural malignant mesothelioma is locally invasive, and progression tends to occur by direct extension of tumor into the pulmonary parenchyma and hilar lymph nodes, the chest wall forming a layer that will compress the lung, the contralateral pleura and other structures within the mediastinum, and through the diaphragm into the abdominal cavity, internal mammary, and supraclavicular lymph nodes, and distant organs via hematogenous dissemination in the later stages of the disease..$^{7-10,16)}$ Tumor extension to the spinal cord is uncommon, and usually occurs by direct extension through the intervertebral foramen or the invaded vertebral body and by hematogenous spread to the surrounding meninges, or rarely by infiltration along the nerve roots. ${ }^{1,15)}$

Received April 17, 2008; Accepted December 26, 2008

Author's present address: H. Okura, M.D., Department of Neurological Surgery, Juntendo University School of Medicine, Tokyo, Japan. 
We report a case of pleural malignant mesothelioma that started with nerve root involvement and subsequently infiltrated the spinal cord.

\section{Case Report}

A 61-year-old man had previous occupational exposure to asbestos while working at a car repair shop dealing with brakes and clutches from 1963 to 1970. Cytologic puncture confirmed the diagnosis of malignant mesothelioma in January 2007. He subsequently underwent right panpleuropneumonectomy under a diagnosis of sarcomatous type mesothelioma in the right pleural space in March 2007. He experienced progressive weakness of the right lower leg from late August 2007, and numbness and weakness of the left leg from early October. He had dysfunction of micturition and defecation at the same time. About one month later the weakness of the right lower leg had occurred, he was ultimately unable to walk due to bilateral lower extremity weakness. He was referred to our department for further examination and treatment, and admitted because magnetic resonance (MR) imaging revealed an intramedullary mass in the thoracic spinal cord.

Neurological examination on admission revealed sensory disturbance, paraparesis, and dysfunction of the bladder and bowels. Sensory deficit was evident at the right $\mathrm{T} 4$ dermatome with decreased touch and pain sensation. Weakness was greater in the right leg than the left with Babinski response. He was hyperreflexic in the lower extremities. Motor function of both legs deteriorated rapidly, resulting in paraplegia, and he was no longer able to leave his bed. Sagittal $\mathrm{T}_{1}$-weighted MR imaging after gadolinium injection showed a heterogeneously enhanced mass in the spinal cord at the T4 level and sagittal $\mathrm{T}_{2}$-weighted $\mathrm{MR}$ imaging showed extensive perifocal edema (Fig. 1A, B). Axial and coronal $\mathrm{T}_{1^{-}}$ weighted MR imaging after gadolinium injection showed no continuity between the thoracic cavity and spinal cord (Fig. 1C, D).

Surgery was performed to confirm the definitive diagnosis on October 24, 2007. The patient was placed in the prone position. Somatosensory evoked potential monitoring revealed diminished amplitude. Right partial laminectomy at T4-T5 was performed through a midline skin incision. The dura mater was not tense. Dural incision exposed the clouded and swollen right posterior nerve root which appeared grayish and soft compared to the left posterior nerve root. The pial surface of the spinal cord was covered by clouded thick arachnoidlike membrane (Fig. 2). No evidence of tumor surrounding the spinal cord intradurally or extradural-
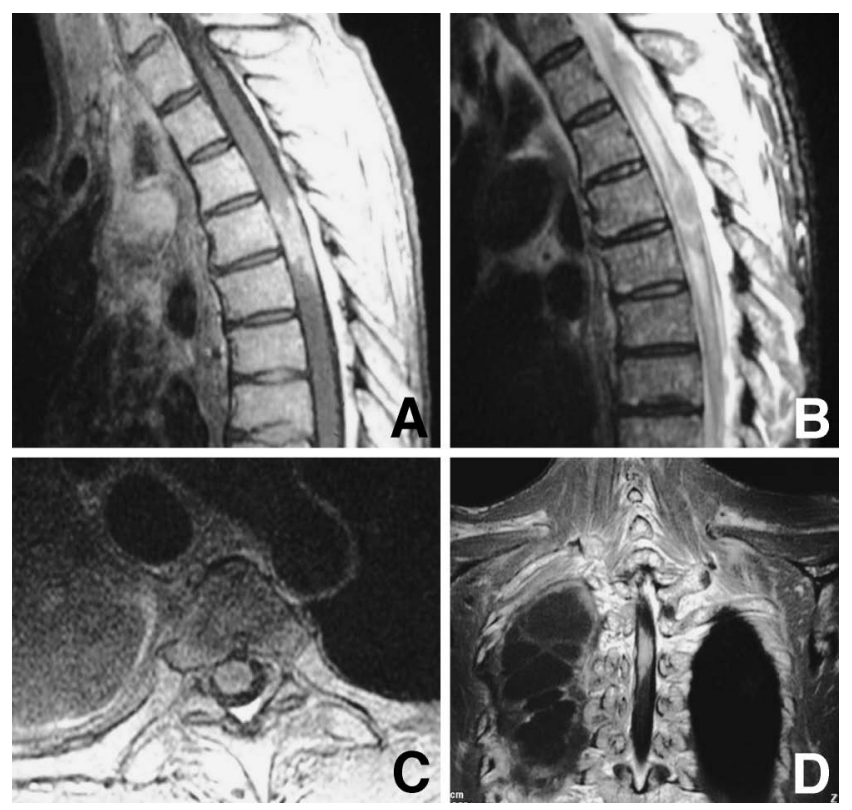

Fig. 1 (A) Sagittal $T_{1}$-weighted magnetic resonance (MR) image of the spine after gadolinium injection showing a heterogeneous irregularly-shaped enhanced mass in the spinal cord at the $\mathrm{T} 4$ level, and (B) sagittal $T_{2}$-weighted MR image of the spine showing extensive peritumoral edema. (C) Axial and (D) coronal $T_{1}$-weighted MR images after gadolinium injection showing no evidence of perimedullary mass.

ly was found. A part of the T4 posterior nerve root with the clouded arachnoid-like membrane was carefully dissected off the spinal cord and sent for histological study. The tumor was identified after making a small myelotomy along the right dorsal root exit zone. The tumor was elastic hard and not so hemorrhagic with internal debulking. A clear plane was difficult to identify between the tumor and surrounding spinal cord. The intramedullary tumor was removed. Complete hemostasis was confirmed by the Valsalva maneuver. Finally, the dura was closed in a watertight fashion. Wound closure was made in the usual manner.

Histological examination of the specimens from the posterior nerve root revealed spindle-shaped neoplastic cells with mitotic activity and pleomorphic nuclei associated with proliferation of fibrous tissue. The T4 nerve root was diffusely infiltrated and completely replaced by the tumor (Fig. 3A). Immunohistochemical staining was strongly positive for keratin (Fig. 3B) and vimentin. These morphological and immunohistochemical features were consistent with a diagnosis of sarcomatous type malignant mesothelioma. 
The postoperative course was favorable. However, his neurological condition remained the same compared to the preoperative state except for slight improvement of sensation around the T4 level. There was no motor function in either leg. He died of respiratory failure 3 months after the diagnosis.

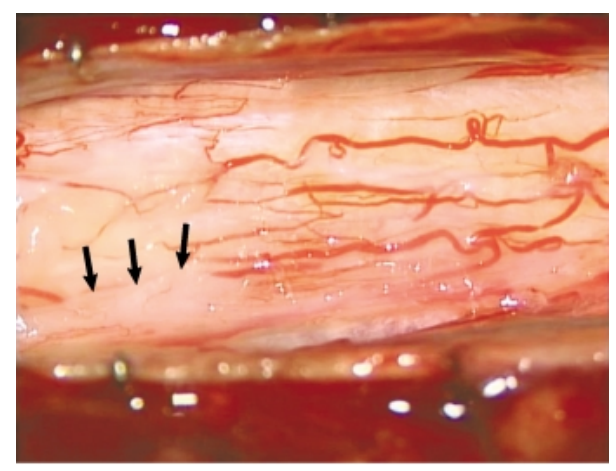

Fig. 2 Intraoperative photograph showing the right posterior nerve root was clouded and swollen (arrows), and pial surface of the spinal cord was covered by clouded thick arachnoid-like membrane.
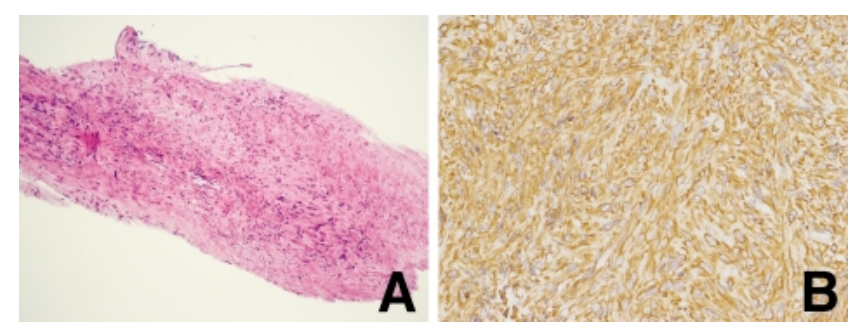

Fig. 3 A: Photomicrograph of the posterior nerve root showing the $\mathrm{T} 4$ nerve root diffusely infiltrated and completely replaced by the tumor. Hematoxylin and eosin stain, original magnification $\times 25$. B: Photomicrograph showing strongly positive immunohistochemical staining for keratin. Original magnification $\times \mathbf{1 0 0}$.

\section{Discussion}

Pleural malignant mesothelioma rarely metastasizes to the nervous system, with only a few cases of pleural malignant mesothelioma involving the spinal canal. ${ }^{7,13,15,19)}$ Infiltration of structures within the spinal canal is very rare, with only 14 reported cases. ${ }^{3,4,7-15,17-20)}$ Initial symptoms are usually spinal pain and paresthesia, and weakness and dysfunction of the bladder and bowels. ${ }^{17)}$ There have been previous case reports of pleural malignant mesothelioma invading the spinal cord through the intervertebral foramen or vertebral body, hematogenous spread via the intercostal arteries or the veins of Batson's plexus, and direct extension along the nerve root.

Autopsy of a 67-year-old man who died shortly after becoming paraplegic found a right thoracic pleural mesothelioma with extensive extradural spread at the T8 level, and diffuse infiltration of the thoracic dura and spinal cord. ${ }^{3)}$ The dura of the thoracic spine was diffusely involved and multiple tumor deposits were found on the arachnoid sheath and within the substance of the cord. Several posterior nerve roots in this region were also thickened. The presence of these multiple discrete foci of mesothelioma deposits were believed to indicate multiple hematogenous metastases spread by the spinal branch of the intercostal arteries.

Spinal cord involvement by pleural malignant mesothelioma has a variety of appearances. There are two distinct patterns of cord infiltration along the nerve root: extension along the pia matter or arachnoid membrane surrounding the nerve root, known as leptomeningeal spread and centrifugal extension into the nerve root, direct invasion. Leptomeningeal spread along the nerve root is interpreted as one of the hematogenous metastases. On the other hand, direct invasion along the nerve root is considered to be neurotropism characterized by the primary tumor. In our patient, the tumor had completely invaded the posterior nerve root, reaching into the spinal cord and forming cord infiltration

Table 1 Clinical characteristics of patients with intramedullary metastatic mesothelioma

\begin{tabular}{|c|c|c|c|c|}
\hline Author (Year) & $\begin{array}{l}\text { Patient's age }(\mathrm{yrs}) / \\
\text { Sex }\end{array}$ & Location & Therapy & Neurological outcome \\
\hline Cooper $(1974)^{3)}$ & $67 / \mathrm{M}$ & $\mathrm{T} 8$ & autopsy findings & not described \\
\hline Hillard et al. $(2007)^{8)}$ & $61 / \mathrm{M}$ & $\mathrm{C} 7-\mathrm{T} 1$ & $\begin{array}{l}\text { extradural decompression, } \\
\text { intradural exploration }\end{array}$ & slight improvement \\
\hline $\begin{array}{l}\text { Payer and von Briel } \\
(2007)^{15)}\end{array}$ & $50 / \mathrm{M}$ & T5 & $\begin{array}{l}\text { extradural decompression, } \\
\text { intradural exploration }\end{array}$ & progressive worsening \\
\hline
\end{tabular}


based on the findings of histological examination. However, the pattern of the extension along the nerve root is difficult to determine.

Intradural involvement is rare, with a few reported cases, ${ }^{4,8,13,19)}$ and intramedullary involvement is extremely rare, with five cases including the present case, all occurring in the thoracic spine (Table 1)..$^{3,8,9,15)}$ Most patients with intramedullary metastatic mesothelioma who underwent surgery did not achieve any improvement in their neurological condition.

Preoperative imaging may not indicate definitively whether the intraspinal involvement is intradural. If the patient suffers neurological deterioration with possible preservable function, neurological decompression with intradural exploration is recommended. Extradural tumor can be removed safely in most cases. However, if intradural tumor is present on the spinal cord, the cleavage plane of the tissue may determine whether successful gross total resection can be achieved. In some cases, partial spinal decompression may be sufficient to prevent further neurological deterioration. ${ }^{8)}$

Radiotherapy and chemotherapy such as cisplatin, irinotecan, doxorubicin, ifosfamide, ${ }^{7)}$ and methotrexate $^{17)}$ have failed to significantly prolong patient survival with a median survival of less than 1 year after symptom onset. ${ }^{5,7,8,10)}$ Recently, management of patients with pleural malignant mesothelioma has used antifolate (pemetrexed or raltitrexed)platinum combinations and new radiotherapy techniques such as intensity-modulated radiation therapy. $^{2)}$

In general, there is no standard treatment for intramedullary spinal cord metastasis. However, there are some reports that radiation therapy only is effective treatment for intramedullary spinal cord metastasis in terms of clinical symptoms and radiological findings. ${ }^{621)}$ In cases with undiagnosed or limited, radioresistant tumors and rapid neurological deterioration, surgical resection is recommended. In our case, we selected surgical resection due to rapidly progressive neurological deterioration and to confirm the definitive diagnosis.

Our case showed no epidural spinal canal or extramedullary involvement, and the tumor appeared to have extended into the spinal cord by directly invading along the $\mathrm{T} 4$ nerve root. Pleural malignant mesothelioma has a tendency to spread along preexisting tissue planes, and this case provides further evidence of the rare ability of pleural mesothelioma to spread along the nerve root, through the intervertebral foramina, and into the spinal cord resulting in neurological sequelae.

We conclude that spinal metastasis must be con- sidered if a patient with pleural malignant mesothelioma shows neurological worsening and MR imaging shows an abnormal lesion in the thoracic spinal cord. However, the patient's neurological condition is very difficult to improve in the presence of intramedullary spinal metastasis. Most patients with pleural malignant mesothelioma ultimately die of respiratory failure.

\section{References}

1) Au VW, Thomas M: Radiological manifestations of malignant pleural mesothelioma. Australas Radiol 47: 111-116, 2003

2) Ceresoli GL, Gridelli C, Santoro A: Multidisciplinary treatment of malignant pleural mesothelioma. Oncologist 12: 850-863, 2007

3) Cooper D: Malignant mesothelioma invading the spinal canal. Postgrad Med J 50(589): 718-723, 1974

4) de Pangher Manzini V, Chizzola A, Brollo A, Bianchi C: [Diffuse neoplastic infiltration of leptomeninges by malignant pleural mesothelioma]. Recenti Prog Med 80: 16-17, 1989 (Ita, with Eng abstract)

5) Edwards JG, Abrams KR, Leverment JN, Spyt TJ, Waller DA, O’Byrne KJ: Prognostic factors for malignant mesothelioma in 142 patients: validation of CALGB and EORTC prognostic scoring systems. Thorax 55: 731-735, 2000

6) Endo S, Hida K, Yano S, Ito M, Yamaguchi S, Kashiwazaki D, Kinoshita R, Shirato H, Iwasaki Y: [Intramedullary spinal cord metastasis treated with radiation therapy: report of 3 cases]. No Shinkei Geka 36: 345-349, 2008 (Jpn, with Eng abstract)

7) Gijtenbeek JM, Brouwer HI, Boerman RH, Wiggenraad RG, Vecht CJ, Smitt PA: Extensive epidural cufflike growth of malignant pleural mesothelioma causing spinal cord compression. J Thorac Cardiovasc Surg 124: 200-202, 2002

8) Hillard VH, Liu JK, Kwok A, Schmidt MH: Perineural spread of malignant mesothelioma resulting in an intradural spinal cord mass: case report. J Neurooncol 81: 185-189, 2007

9) Lee AW, Nikitins I, Pozza C, Koblar SA: Pleural mesothelioma with extension into the thoracic spinal cord. Intern Med J 35: 195-196, 2005

10) Lee YC, Light RW, Musk AW: Management of malignant pleural mesothelioma: a critical review. Curr Opin Pulm Med 6: 267-274, 2000

11) Margery J, Grassin F, Le Moulec S, Ruffié P: [Spinal cord compression from a malignant pleural mesothelioma]. Rev Pneumol Clin 61: 112-114, 2005 (Fre, with Eng abstract)

12) Mikhael MA, Paige ML, Thayer C: Computerized tomography of malignant pleural mesothelioma with spinal canal invasion. Comput Radiol 6: 11-15, 1982

13) Murray JB, Neilly JB, Hadley D, Moran F, McKean M: Diffuse meningeal thickening associated with pleural mesothelioma. Thorax 45: 70-71, 1990 
14) Nixon PF, Wolfenden WH, Hirst E: Paraplegia and destruction of ribs. Med J Aust 1: 207-212, 1964

15) Payer $M$, von Briel $T$ : Intradural pleural malignant mesothelioma. Acta Neurochir (Wien) 149: 1053-1056, 2007

16) Robinson BW, Musk AW, Lake RA: Malignant mesothelioma. Lancet 366(9483): 397-408, 2005

17) Rojas JL, Alfageme I, De la Cruz I, Reyes N, Muñoz J: Radicular involvement and medullary invasion from a malignant mesothelioma. Respiration 68: 106-108, 2001

18) Semb G: Diffuse malignant pleural mesothelioma: a clinicopathological study of 10 fatal cases. Acta Chir Scand 126: 78-91, 1963

19) Steel TR, Allibone J, Revesz T, D’Arrigo C, Crockard HA: Intradural neurotropic spread of malignant mesothelioma. Case report and review of the litera- ture. J Neurosurg 88: 122-125, 1998

20) Taryle DA, Lakshminarayan S, Sahn SA: Pleural mesotheliomas - an analysis of 18 cases and review of the literature. Medicine (Baltimore) 55: 153-162, 1976

21) Villegas AE, Guthrie TH: Intramedullary spinal cord metastasis in breast cancer: clinical features, diagnosis, and therapeutic consideration. Breast $J$ 10: 532-535, 2004

Address reprint requests to: Hidehiro Okura, M.D., Department of Neurological Surgery, Juntendo University School of Medicine, 2-1-1 Hongo, Bunkyo-ku, Tokyo 113-8421, Japan.

e-mail: hohkura@juntendo.ac.jp 